Research Article

Open Access

Special Issue Dedicated to Charles R. Johnson

C. Marijuán*, M. Pisonero, and Ricardo L. Soto

\title{
Updating a map of sufficient conditions for the real nonnegative inverse eigenvalue problem
}

https://doi.org/10.1515/spma-2019-0018

Received July 29, 2019; accepted November 28, 2019

\begin{abstract}
The real nonnegative inverse eigenvalue problem (RNIEP) asks for necessary and sufficient conditions in order that a list of real numbers be the spectrum of a nonnegative real matrix. A number of sufficient conditions for the existence of such a matrix are known. The authors gave in [11] a map of sufficient conditions establishing inclusion relations or independency relations between them. Since then new sufficient conditions for the RNIEP have appeared. In this paper we complete and update the map given in [11].
\end{abstract}

Keywords: real nonnegative inverse eigenvalue problem, sufficient conditions, nonnegative matrices

Classification: 15A29; 15A18; 15B51

\section{Introduction}

The real nonnegative inverse eigenvalue problem (hereafter RNIEP) is the problem of characterizing all possible real spectra of entrywise nonnegative matrices. This problem remains unsolved. A complete solution is known only for spectra of size $n \leq 4$.

A number of realizability criteria or sufficient conditions for the existence of a nonnegative matrix with a given real spectrum have been obtained, from different points of view. In [11] the authors construct a map of sufficient conditions for the RNIEP, in which they show inclusion or independency relations between these conditions. However, a pair of questions were left unsolved and the answers are now known: Soto 2 criterion implies Perfect $2^{+}$criterion and the inclusion of Perfect $2^{+}$criterion in Soto-Rojo criterion is strict.

Since 2007 new sufficient conditions for the RNIEP have appeared, [3, 14]. In this paper we discuss new relations of inclusion or independency between these new sufficient conditions and the previous ones studied in [11].

The paper is organized as follows: Section 2 contains the list of all sufficient conditions that we shall consider, in chronological order, and some technical results that we will use in the next section. Section 3 completes and updates the map given in [11] for the RNIEP.

\footnotetext{
^Corresponding Author: C. Marijuán: Dpto. Matemática Aplicada, E.T.S.I. Informática, Paseo de Belén 15, 47011-Valladolid, Spain, E-mail: marijuan@mat.uva.es

M. Pisonero: Dpto. Matemática Aplicada, E.T.S. de Arquitectura, Avenida de Salamanca 18, 47014-Valladolid, Spain, E-mail: mpisoner@maf.uva.es

Ricardo L. Soto: Dpto. de Matemáticas, Universidad Católica del Norte, Antofagasta, Chile, E-mail: rsoto@ucn.cl
} 


\section{Sufficient conditions for the RNIEP}

In this paper, by a list we understand a collection $\Lambda=\left\{\lambda_{1}, \ldots, \lambda_{n}\right\}$ of real numbers with possible repetitions. By a partition of a list $\Lambda$ we mean a family of sublists of $\Lambda$ whose disjoint union is $\Lambda$. As is commonly accepted, we understand that a summatory is equal to zero when the index set of the summatory is empty.

We will say that a list $\Lambda$ is (symmetrically) realizable if it is the spectrum of an entrywise (symmetric) nonnegative matrix $A$. In this case $A$ is said to be a realizing matrix.

The RNIEP has an obvious solution when only nonnegative real numbers are considered, so the interest of the problem is when there is at least one negative number in the list.

In what follows we list the sufficient conditions, or realizability criteria, that we are going to consider, in chronological order. The first result in this area was announced by Suleǐmanova in 1949 and proved by Perfect in 1953.

Theorem 1. (Perfect $2[12], 1955)$ Let $\left\{\lambda_{0}, \lambda_{1}, \ldots, \lambda_{r}\right\}$, with $\lambda_{0} \geq\left|\lambda_{i}\right|$ for $i=1, \ldots, r$, be realizable by a matrix with diagonal elements $\omega_{0}, \omega_{1}, \ldots, \omega_{r}$ and let $\Lambda=\left\{\lambda_{0}, \lambda_{1}, \ldots, \lambda_{r}, \lambda_{r+1}, \ldots, \lambda_{n}\right\}$ with $-\lambda_{0} \leq \lambda_{i} \leq 0$ for $i=$ $r+1, \ldots, n$. If there exists a partition $\left\{\lambda_{01}, \ldots, \lambda_{0 t_{0}}\right\} \cup\left\{\lambda_{11}, \ldots, \lambda_{1 t_{1}}\right\} \cup \cdots \cup\left\{\lambda_{r 1}, \ldots, \lambda_{r t_{r}}\right\}$ (some or all of the lists may be empty) of $\left\{\lambda_{r+1}, \ldots \lambda_{n}\right\}$ such that

$$
\omega_{i}+\sum_{j=1}^{t_{i}} \lambda_{i j} \geq 0 \quad \text { for } \quad i=0,1, \ldots, r
$$

then $\Lambda$ is realizable.

Although Perfect gives the previous theorem for stochastic matrices, the normal form of a stochastic matrix allows us to give the theorem for the nonnegative case. Note that originally the $w_{i}$ 's are diagonal elements of a stochastic matrix. In fact, the realizing matrix with diagonal elements $\omega_{0}, \omega_{1}, \ldots, \omega_{r}$ can be taken with all its row sums equal to $\lambda_{0}$ (see Lemma 2). When in the previous theorem the elements of the list $\left\{\lambda_{0}, \lambda_{1}, \ldots, \lambda_{r}\right\}$ are all nonnegative there always exists a realization of this list. We will call this condition Perfect $2^{+}$, i.e. Theorem 1 when $\lambda_{i} \geq 0$ for $i=0,1, \ldots, r$. (See [12, Theorem 3])

The previous theorem has a constructive proof, in the sense that it allows us to construct a realizing matrix. Brauer's theorem [4], which allows only one eigenvalue of a complex matrix to be modified without changing the others, plays a fundamental role in this construction.

Theorem 2. (Brauer [4], 1952) Let $A$ be a complex matrix with spectrum $\left\{\lambda_{1}, \ldots, \lambda_{n}\right\}$. Let $k \in\{1, \ldots, n\}$, $x=\left(x_{1}, \ldots, x_{n}\right)^{t}$ be an eigenvector associated to $\lambda_{k}$ and $q$ any $n$-dimensional vector. Then the matrix $A+x q^{t}$ has spectrum $\left\{\lambda_{1}, \ldots, \lambda_{k-1}, \lambda_{k}+x^{t} q, \lambda_{k+1}, \ldots, \lambda_{n}\right\}$.

In order to make use of Theorem 1, Perfect [12] gives sufficient conditions under which $\lambda_{0}, \lambda_{1}, \ldots, \lambda_{r}$ and $\omega_{0}, \omega_{1}, \ldots, \omega_{r}$ are the eigenvalues and the diagonal elements, respectively, of a nonnegative matrix.

Lemma 1. Let $\Lambda=\left\{\lambda_{1}, \ldots, \lambda_{r}\right\}$, with $\lambda_{1} \geq|\lambda|$ for $\lambda \in \Lambda$, be realizable. The real numbers $\omega_{1}, \ldots, \omega_{r}$ are the diagonal elements of a nonnegative matrix with spectrum $\Lambda$ if

i) $0 \leq \omega_{i} \leq \lambda_{1}$, for $i=1, \ldots, r$;

ii) $\omega_{1}+\cdots+\omega_{r}=\lambda_{1}+\cdots+\lambda_{r}$;

iii) $\omega_{i} \geq \lambda_{i}$ and $\omega_{1} \geq \lambda_{i}$, for $i=2, \ldots, r$.

For $r=1$ and $r=2$ Perfect [12] gives necessary and sufficient conditions. The following lemma recalls the result described in the introduction of [7] about the equivalence of the row stochastic and the nonnegative eigenvalue problems. We include the proof of this result here to show that the diagonal elements are preserved. This lemma will be required for Section 3 (theorems 9 and 10). 
Lemma 2. Every nonnegative matrix $A$ with spectral radius $\rho$ is cospectral with a nonnegative matrix $B$ preserving the diagonal elements of $A$ and all its row sums equal to $\rho$. Furthermore, if $A$ has a positive eigenvector associated to $\rho$, then $B$ is similar to $A$.

Proof. Let $A=\left(a_{i j}\right)$ be a nonnegative matrix with a positive eigenvector $x=\left(x_{1}, \ldots, x_{n}\right)^{t}$ associated to $\rho$ and let $D$ be the diagonal matrix whose $(i, i)$ element is $x_{i}$. Then the matrix

$$
B=D^{-1} A D=\left(\frac{x_{j}}{x_{i}} a_{i j}\right)
$$

is nonnegative, is similar to $A$ and has the same diagonal elements as $A$. The matrix $B$ has all its row sums equal to $\rho$ because $e=(1, \ldots, 1)^{t}$ is an eigenvector of $B$ associated to $\rho$ :

$$
\left.\begin{array}{c}
A x=\rho x \\
D e=x
\end{array}\right\} \Rightarrow B e=D^{-1} A D e=D^{-1} A x=\rho D^{-1} x=\rho e .
$$

Now let $A=\left(a_{i j}\right)$ be an arbitrary nonnegative matrix, then $A$ is permutationally similar to

$$
P^{t} A P=\left(\begin{array}{ccccc}
A_{11} & 0 & \cdots & \cdots & 0 \\
A_{21} & A_{22} & 0 & \cdots & 0 \\
\vdots & \vdots & \ddots & \ddots & \vdots \\
\vdots & \vdots & \vdots & \ddots & 0 \\
A_{k 1} & A_{k 2} & \cdots & \cdots & A_{k k}
\end{array}\right)
$$

with $A_{i i}$ irreducible or 1-by-1 and zero, for $i=1, \ldots, k$. Let $\rho_{i}$ be the spectral radius of $A_{i i}$. Any irreducible matrix has a positive eigenvector associated to its spectral radius. Let $B_{i i}$ be the nonnegative matrix obtained before similar to $A_{i i}$ which preserves the diagonal elements of $A_{i i}$ and all its row sums equal to $\rho_{i}$ if $A_{i i}$ is irreducible and 0 otherwise. Reindexing the matrices $\left\{B_{i i}\right\}_{i=1}^{k}$, if necessary, we can suppose $\rho=\rho_{1}$. Let $B_{i 1}$ be the matrix with the same number of columns as $B_{11}$ and the same number of rows as $B_{i i}$, for $i=2, \ldots, k$, with all the elements of the first column equal to the nonnegative number $\rho-\rho_{i}$ and the other columns equal to zero. Then the matrix

$$
B=\left(\begin{array}{ccccc}
B_{11} & 0 & \cdots & \cdots & 0 \\
B_{21} & B_{22} & 0 & \cdots & 0 \\
\vdots & 0 & \ddots & \ddots & \vdots \\
\vdots & \vdots & \ddots & \ddots & 0 \\
B_{k 1} & 0 & \cdots & 0 & B_{k k}
\end{array}\right)
$$

satisfies the lemma.

Theorem 3. (Kellogg [8], 1971) Let $\Lambda=\left\{\lambda_{0}, \lambda_{1}, \ldots, \lambda_{n}\right\}$ with $\lambda_{0} \geq|\lambda|$ for $\lambda \in \Lambda$ and $\lambda_{i} \geq \lambda_{i+1}$ for $i=0, \ldots, n-1$. Let $M$ be the greatest index $j(0 \leq j \leq n)$ for which $\lambda_{j} \geq 0$ and $K=\left\{i \in\{1, \ldots,\lfloor n / 2\rfloor\} / \lambda_{i} \geq 0, \lambda_{i}+\lambda_{n+1-i}<0\right\}$. If

$$
\lambda_{0}+\sum_{i \in K, i<k}\left(\lambda_{i}+\lambda_{n+1-i}\right)+\lambda_{n+1-k} \geq 0 \text { for all } k \in K,
$$

and

$$
\lambda_{0}+\sum_{i \in K}\left(\lambda_{i}+\lambda_{n+1-i}\right)+\sum_{j=M+1}^{n-M} \lambda_{j} \geq 0
$$

then $\Lambda$ is realizable. 
Theorem 4. (Borobia [1], 1995) Let $\Lambda=\left\{\lambda_{0}, \lambda_{1}, \ldots, \lambda_{n}\right\}$ with $\lambda_{i} \geq \lambda_{i+1}$ for $i=0, \ldots, n-1$ and let $M$ be the greatest index $j(0 \leq j \leq n)$ for which $\lambda_{j} \geq 0$. If there exists a partition $J_{1} \cup \cdots \cup J_{t}$ of $\left\{\lambda_{M+1}, \ldots, \lambda_{n}\right\}$ such that

$$
\lambda_{0} \geq \lambda_{1} \geq \cdots \geq \lambda_{M}>\sum_{\lambda \in J_{1}} \lambda \geq \cdots \geq \sum_{\lambda \in J_{t}} \lambda
$$

satisfies the Kellogg condition, then $\Lambda$ is realizable.

Let $\mathcal{K}$ be a realizability criterion. If a list of real numbers satisfies $\mathcal{K}$ we say that the list is $\mathcal{K}$ realizable. We denote the set of $\mathcal{K}$ realizable lists as

$$
\mathcal{R}_{\mathcal{K}}=\{\Lambda \subset \mathbb{R}: \Lambda \text { is } \mathcal{K} \text { realizable }\} .
$$

In this paper $\mathcal{K}$ will be the surname of an author(s). For example, a list satisfying Theorem 4 will be said Borobia realizable.

Theorem 5. (Soto 2 [13], 2003) Let $\Lambda$ be a list that admits a partition

$$
\left\{\lambda_{11}, \ldots, \lambda_{1 t_{1}}\right\} \cup \cdots \cup\left\{\lambda_{r 1}, \ldots, \lambda_{r t_{r}}\right\}
$$

with $\lambda_{11} \geq|\lambda|$ for $\lambda \in \Lambda, \lambda_{i j} \geq \lambda_{i, j+1}$ and $\lambda_{i 1} \geq 0$ for $i=1, \ldots, r$ and $j=1, \ldots, t_{i}$. For each list $\left\{\lambda_{i 1}, \ldots, \lambda_{i t_{i}}\right\}$ of the partition we define $S_{i}$ as

$$
\begin{gathered}
S_{i j}=\lambda_{i j}+\lambda_{i, t_{i}-j+1} \text { for } j=2, \ldots,\left\lfloor t_{i} / 2\right\rfloor \\
S_{i,\left(t_{i}+1\right) / 2}=\min \left\{\lambda_{i,\left(t_{i}+1\right) / 2}, 0\right\} \quad \text { if } \quad t_{i} \quad \text { is odd for } \quad i=1, \ldots, r .
\end{gathered}
$$

Let

$$
T_{i}=\lambda_{i 1}+\lambda_{i t_{i}}+\sum_{S_{i j}<0} S_{i j} \quad \text { for } \quad i=1, \ldots, r
$$

and

$$
L=\max \left\{-\lambda_{1 t_{1}}-\sum_{S_{1 j}<0} S_{1 j}, \max _{2 \leq i \leq r}\left\{\lambda_{i 1}\right\}\right\} .
$$

If

$$
\lambda_{11} \geq L-\sum_{T_{i}<0,2 \leq i \leq r} T_{i}
$$

then $\Lambda$ is realizable.

Theorem 6. (Soto-Rojo [15], 2006) Let $\Lambda$ be a list that admits a partition

$$
\left\{\lambda_{11}, \ldots, \lambda_{1 t_{1}}\right\} \cup \cdots \cup\left\{\lambda_{s 1}, \ldots, \lambda_{s t_{s}}\right\}
$$

with $\lambda_{11} \geq|\lambda|$ for $\lambda \in \Lambda, \lambda_{i j} \geq \lambda_{i, j+1}$ and $\lambda_{i 1} \geq 0$ for $i=1, \ldots$, s and $j=1, \ldots, t_{i}$. Let $\omega_{1}, \ldots, \omega_{s}$ be nonnegative numbers such that there exists a $s \times s$ nonnegative matrix $B$ with eigenvalues $\lambda_{11}, \lambda_{21}, \ldots, \lambda_{s 1}$ and diagonal entries $\omega_{1}, \ldots, \omega_{s}$. If the lists $\left\{\omega_{i}, \lambda_{i 2}, \ldots, \lambda_{i t_{i}}\right\}$ with $\omega_{i} \geq \lambda_{i 2}$, for $i=1, \ldots, s$, are realizable, then $\Lambda$ is realizable.

By its own nature, the Soto-Rojo criterion trivially contains any sufficient condition: if a list $\Lambda$ is realizable, then it is Soto-Rojo realizable with $s=1$ and $w_{1}$ equal to the spectral radius of $\Lambda$. There exist in the literature some other criteria of the same nature. Their interest lies in providing different procedures to realize certain lists. For example, Soto-Rojo-Manzaneda [16], 2011: which is Soto-Rojo with $\lambda_{11}, \lambda_{21}, \ldots, \lambda_{s 1}$ not necessary nonnegative. It also has a symmetric version.

The sufficient conditions of Soto 2 and Soto-Rojo have constructive proofs, which allow us to compute an explicit realizing matrix. Both use Brauer's theorem for the construction of a realizing matrix.

In [3] the authors define a special kind of realizability which is closely related to the idea of "negativity compensation". This criterion is based on the following three results: 
- Rule 1: Let $\Lambda=\left\{\lambda_{1}, \lambda_{2}, \ldots, \lambda_{n}\right\} \subset \mathbb{C}$ be a realizable list with $\lambda_{1} \geq|\lambda|$ for $\lambda \in \Lambda$ and let $\lambda_{2}$ be real. Then for every $\epsilon>0$ the lists $\left\{\lambda_{1}+\epsilon, \lambda_{2}+\epsilon, \lambda_{3}, \ldots, \lambda_{n}\right\}$ and $\left\{\lambda_{1}+\epsilon, \lambda_{2}-\epsilon, \lambda_{3}, \ldots, \lambda_{n}\right\}$, are also realizable. (See Guo [6])

- Rule 2 : Let $\Lambda=\left\{\lambda_{1}, \lambda_{2}, \ldots, \lambda_{n}\right\}$ be a realizable list with $\lambda_{1} \geq|\lambda|$ for $\lambda \in \Lambda$ and let $\epsilon>0$. Then $\left\{\lambda_{1}+\right.$ $\left.\epsilon, \lambda_{2}, \ldots, \lambda_{n}\right\}$ is also realizable.

- Rule 3 : Let $\Lambda_{1}$ and $\Lambda_{2}$ be realizable lists. Then the list $\Lambda_{1} \cup \Lambda_{2}$ is realizable.

Theorem 7. (Borobia-Moro-Soto [3], 2008) Let $\Lambda=\left\{\lambda_{1}, \lambda_{2}, \ldots, \lambda_{n}\right\}$ be a list of real numbers. If $\Lambda$ can be reached starting from the $n$ realizable lists

$$
\{0\},\{0\}, \ldots,\{0\}
$$

successively applying, in any order and any number of times, either Rule 1, Rule 2 or Rule 3, then $\Lambda$ is realizable.

The previous theorem, in [3], is given as the concept of $C$-realizability. As we will see later, Rule 1 can be relaxed to:

- Rule $1^{*}$ : Rule 1 except the case $\left\{\lambda_{1}+\epsilon, \lambda_{2}-\epsilon, \lambda_{3}, \ldots, \lambda_{n}\right\}$ with $\lambda_{2}>0$ and $\lambda_{2}-\epsilon<0$.

We define the game criterion as the variation of Theorem 7 obtained by changing Rule 1 to Rule $1^{\star}$. The $C$ realizability criterion was originally introduced in terms of a game with tokens to put inside of an empty deposit. This rule has been slightly modified here, because the compensation is used for $\lambda_{2} \leq 0$, see comments after Theorem 1.1 in [3].

Let $\mathcal{K}$ be a realizability criterion. Following the definitions in [2, Section 4] we define the $\mathcal{K}$ negativity of a list $\Lambda=\left\{\lambda_{1}, \lambda_{2}, \ldots, \lambda_{n}\right\}$ of real numbers, with $\lambda_{1} \geq \lambda_{j}$ for $j=2, \ldots, n$, as:

$$
\mathcal{N}_{\mathcal{K}}(\Lambda)= \begin{cases}+\infty & \text { if }\left\{\lambda_{1}+\delta, \lambda_{2}, \ldots, \lambda_{n}\right\} \text { is } \\ & \text { not } \mathcal{K} \text { realizable } \forall \delta \geq 0 \\ \min \left\{\delta \geq 0:\left\{\lambda_{1}+\delta, \lambda_{2}, \ldots, \lambda_{n}\right\} \text { is } \mathcal{K} \text { realizable }\right\} & \text { otherwise }\end{cases}
$$

and when $\Lambda$ is $\mathcal{K}$ realizable we define the $\mathcal{K}$ realizability margin of $\Lambda$ as the number:

$$
\mathcal{M}_{\mathcal{K}}(\Lambda)=\max \left\{\epsilon \geq 0: \begin{array}{c}
\left\{\lambda_{1}-\epsilon, \lambda_{2}, \ldots, \lambda_{n}\right\} \text { is } \mathcal{K} \text { realizable } \\
\text { and } \lambda_{1}-\epsilon \geq\left|\lambda_{j}\right| \text { for } j=2, \ldots, n
\end{array}\right\} .
$$

Note that the $\mathcal{K}$ negativity of a list measures, in a certain sense, how far the list is from being $\mathcal{K}$ realizable. A similar interpretation can be given for the concept of $\mathcal{K}$ realizability margin of a $\mathcal{K}$ realizable list. For properties, closed expressions or bounds of these concepts see [10].

Theorem 8. (Soto $\mathrm{p}[14], 2013)$ Let $p$ be an integer with $p \geq 2$. Let $\Lambda$ be a list that admits a partition

$$
\left\{\lambda_{11}, \ldots, \lambda_{1 t_{1}}\right\} \cup \cdots \cup\left\{\lambda_{r 1}, \ldots, \lambda_{r t_{r}}\right\}
$$

with $\lambda_{11} \geq|\lambda|$ for $\lambda \in \Lambda, \lambda_{i j} \geq \lambda_{i, j+1}$ and $\lambda_{i 1} \geq 0$ for $i=1, \ldots, r$ and $j=1, \ldots, t_{i}$, and $\left\{\lambda_{11}, \ldots, \lambda_{1 t_{1}}\right\}$ Soto $p-1$ realizable with $p \geq 3$. Let $\mathcal{N}_{S p-1}\left(\Lambda_{i}\right)$ be the Soto $p$-1 negativity of $\Lambda_{i}=\left\{\lambda_{i 1}, \ldots, \lambda_{i t_{i}}\right\}$ and $\mathcal{M}_{S p-1}\left(\Lambda_{i}\right)$ the Soto $p-1$ realizability margin of $\Lambda_{i}$. Let

$$
\gamma=\max \left\{\lambda_{11}-\mathcal{M}_{S p-1}\left(\Lambda_{1}\right), \max _{2 \leq i \leq r}\left\{\lambda_{i 1}\right\}\right\} .
$$

If

$$
\lambda_{11} \geq \gamma+\sum_{\Lambda_{i} \notin \mathcal{R}_{S p-1}} \mathcal{N}_{S p-1}\left(\Lambda_{i}\right),
$$

then $\Lambda$ is (simmetrically) realizable. 


\section{Updating the inclusion relations for the RNIEP}

In this section we update the nonnegative map given in [11] for the RNIEP:

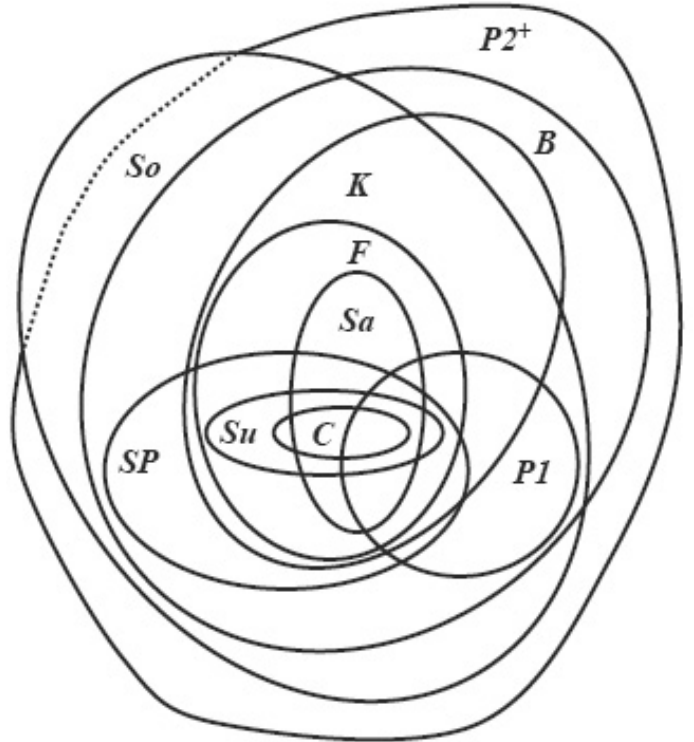

Sulě̌manova $=S u$

Suleǐmanova-Perfect $=S P$

Perfect $1=P 1$

Perfect $2^{+}=P 2^{+}$

Ciarlet $=C$

Kellogg $=K$

Salzmann $=S a$

Fiedler $=$ Soto $1=F$

Borobia $=B$

Soto $2=$ So

- Perfect $2^{+}$does not imply Soto 2 and the dotted line on the map expresses that we did not know if Soto 2 implies (or not) Perfect $2^{+}$. Now we know that Soto 2 implies Perfect $2^{+}$.

- Soto-Rojo contains all the realizability criteria in the previous map, and now we know that the inclusion of Perfect $2^{+}$, for $r \geq 1$, in Soto-Rojo, for $s \geq 2$, is strict (see Theorem 10). Note that we exclude Perfect $2^{+}$for $r=0$ because it is Suleǐmanova, and we exclude Soto-Rojo for $s=1$ because, as was commented before, this trivially includes any sufficient condition.

Therefore, to update the inclusion relations for the RNIEP, we need to place the game and Soto $\mathrm{p}$ sufficient conditions in the map. In [3], the authors prove that the realizability criteria Kellogg, Borobia and Soto 2 imply the $C$-realizability criterion and, moreover, that the inclusions are strict. Going through the proofs in [3], it is easy to see that the authors prove that Kellogg, Borobia and Soto 2 imply the game criterion (theorems 3.2, 3.5 and 3.7). This is due to the fact that any time Rule 1 appears, it is used as Rule $1^{\star}$.

The same argument used in [3], to prove that Soto 2 implies game, can be used to prove that Soto $p$, with $p \geq 3$, also implies game.

Theorem 9. Game implies Perfect $2^{+}$and the inclusion is strict.

Proof. We will prove that Rule $1^{\star}$, Rule 2 and Rule 3 are closed under Perfect $2^{+}$criterion. It is clear that Rule 3 preserves Perfect $2^{+}$: the union of two Perfect $2^{+}$realizable spectra is a Perfect $2^{+}$realizable spectrum. Let us see that rules 2 and $1^{\star}$ also preserve this sufficient condition.

Let

$$
\Lambda=\left\{\lambda_{0}, \lambda_{1}, \ldots, \lambda_{r}, \lambda_{r+1}, \ldots, \lambda_{n}\right\}
$$

satisfy Perfect $2^{+}: \lambda_{0} \geq \lambda_{i} \geq 0$ and $-\lambda_{0} \leq \lambda_{j} \leq 0$ for $i=1, \ldots, r$ and $j=r+1, \ldots, n,\left\{\lambda_{0}, \lambda_{1}, \ldots, \lambda_{r}\right\}$ realizable by a matrix $A$ with diagonal elements $\omega_{0}, \omega_{1}, \ldots, \omega_{r}$ and there exists a partition $\left\{\lambda_{01}, \ldots, \lambda_{0 t_{0}}\right\} \cup$ $\left\{\lambda_{11}, \ldots, \lambda_{1 t_{1}}\right\} \cup \cdots \cup\left\{\lambda_{r 1}, \ldots, \lambda_{r t_{r}}\right\}$ of $\left\{\lambda_{r+1}, \ldots \lambda_{n}\right\}$ such that

$$
\omega_{i}+\sum_{j=1}^{t_{i}} \lambda_{i j} \geq 0 \quad \text { for } \quad i=0,1, \ldots, r .
$$

From Lemma 2 we can take $A$ with all its row sums equal to $\lambda_{0}$. Then $e=(1, \ldots, 1)^{t}$ is an eigenvector of $A$ associated to $\lambda_{0}$. Let $\varepsilon>0$ and prove: 
a) $\Lambda_{\varepsilon}=\left\{\lambda_{0}+\varepsilon, \lambda_{1}, \ldots, \lambda_{r}, \lambda_{r+1}, \ldots, \lambda_{n}\right\}$ is Perfect $2^{+}$realizable.

Let $q=(\varepsilon, 0, \ldots, 0)^{t}$. Note that the matrix

$$
A_{\varepsilon}=A+e q^{t}=A+\left(\begin{array}{cccc}
\varepsilon & 0 & \cdots & 0 \\
\vdots & \vdots & \ddots & \vdots \\
\varepsilon & 0 & \cdots & 0
\end{array}\right)
$$

is nonnegative with diagonal elements $\omega_{0}+\varepsilon, \omega_{1}, \ldots, \omega_{r}$ and all its row sums equal to $\lambda_{0}+\varepsilon$. Brauer's theorem guarantees that the spectrum of $A_{\varepsilon}$ is $\left\{\lambda_{0}+e^{t} q, \lambda_{1}, \ldots, \lambda_{r}\right\}=\left\{\lambda_{0}+\varepsilon, \lambda_{1}, \ldots, \lambda_{r}\right\}$. Hence $\Lambda_{\varepsilon}$ satisfies Perfect $2^{+}$with $A_{\varepsilon}$ and the same partition of $\left\{\lambda_{r+1}, \ldots \lambda_{n}\right\}$ as $\Lambda$.

b) $\Lambda_{\varepsilon}=\left\{\lambda_{0}+\varepsilon, \lambda_{1}, \ldots, \lambda_{r}, \lambda_{r+1}+\varepsilon, \lambda_{r+2}, \ldots, \lambda_{n}\right\}$, with $\lambda_{r+1}+\varepsilon \leq 0$, is Perfect $2^{+}$realizable.

The same matrix $A_{\varepsilon}$ as in statement a) and the "same" partition of $\left\{\lambda_{r+1}+\varepsilon, \lambda_{r+2}, \ldots \lambda_{n}\right\}$ as $\Lambda$ show that $\Lambda_{\varepsilon}$ satisfies Perfect $2^{+}$.

c) $\Lambda_{\varepsilon}=\left\{\lambda_{0}+\varepsilon, \lambda_{1}, \ldots, \lambda_{r}, \lambda_{r+1}+\varepsilon, \lambda_{r+2}, \ldots, \lambda_{n}\right\}$, with $\lambda_{r+1}+\varepsilon>0$, is Perfect $2^{+}$realizable.

Let $q=(\varepsilon, 0, \ldots, 0)^{t}$. Hence $\Lambda_{\varepsilon}$ satisfies Perfect $2^{+}$with

$$
A_{\varepsilon}=\left(\begin{array}{cc}
A+e q^{t} & 0 \\
0 & \lambda_{r+1}+\varepsilon
\end{array}\right)
$$

and the induced partition of $\left\{\lambda_{r+2}, \ldots \lambda_{n}\right\}$ from $\left\{\lambda_{r+1}, \ldots \lambda_{n}\right\}$ as part of $\Lambda$

d) $\Lambda_{\varepsilon}=\left\{\lambda_{0}+\varepsilon, \lambda_{1}+\varepsilon, \lambda_{2}, \ldots, \lambda_{r}, \lambda_{r+1}, \ldots, \lambda_{n}\right\}$ is Perfect $2^{+}$realizable.

Case $\lambda_{1} \neq \lambda_{0}$. Let $x=\left(x_{0}, x_{1}, \ldots, x_{r}\right)^{t}$ be an eigenvector of $A$ associated to $\lambda_{1}$ and let $j_{\max }$ and $j_{\min }$ be the smallest indices with

$$
x_{j_{\max }}=\max \left\{x_{0}, x_{1}, \ldots, x_{r}\right\}=a \quad \text { and } \quad x_{j_{\min }}=\min \left\{x_{0}, x_{1}, \ldots, x_{r}\right\}=b,
$$

respectively. Note that $a>b$, because if they were equal then $x=a e$ would be an eigenvector associated to the two different eigenvalues $\lambda_{0}$ and $\lambda_{1}$ of $A$. Without loss of generality we can assume $j_{\max }<j_{\min }$. Let $q=\left(q_{0}, q_{1}, \ldots, q_{r}\right)^{t}$ and $p=\left(p_{0}, p_{1}, \ldots, p_{r}\right)^{t}$ be defined by

$$
q_{i}=\left\{\begin{array}{ll}
\frac{-b \varepsilon}{a-b} & \text { if } \quad i=j_{\max }, \\
\frac{a \varepsilon}{a-b} & \text { if } \quad i=j_{\min }, \\
0 & \text { if } \quad i \neq j_{\max }, j_{\min }
\end{array} \quad \text { and } p_{i}=\left\{\begin{array}{lll}
\frac{\varepsilon}{a-b} & \text { if } \quad i=j_{\max }, \\
\frac{-\varepsilon}{a-b} & \text { if } \quad i=j_{\min }, \\
0 & \text { if } \quad i \neq j_{\max }, j_{\min } .
\end{array}\right.\right.
$$

Observe that the matrix $A_{\varepsilon}=A+x p^{t}+e q^{t}$

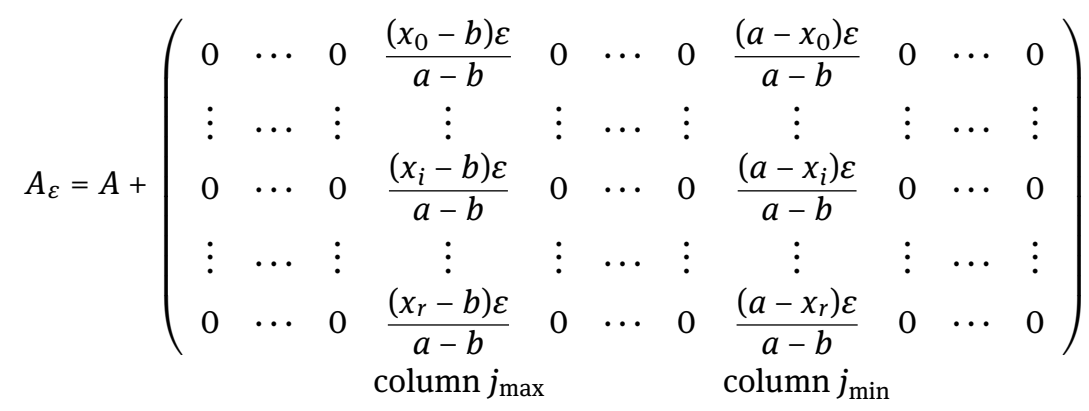

is nonnegative with diagonal elements

$$
\omega_{0}, \omega_{1}, \ldots, \omega_{j_{\max }-1}, \omega_{j_{\max }}+\varepsilon, \omega_{j_{\max }+1}, \ldots, \omega_{j_{\min }-1}, \omega_{j_{\min }}+\varepsilon, \omega_{j_{\min }+1}, \ldots, \omega_{r}
$$

and all its row sums equal to $\lambda_{0}+\varepsilon$. The application of Brauer's theorem twice guarantees that the spectrum of $A_{\varepsilon}$ is $\left\{\lambda_{0}+e^{t} q, \lambda_{1}+x^{t} p, \lambda_{2}, \ldots, \lambda_{r}\right\}=\left\{\lambda_{0}+\varepsilon, \lambda_{1}+\varepsilon, \lambda_{2}, \ldots, \lambda_{r}\right\}$ : 
First, the spectrum of

$$
A+x p^{t}=A+\left(\begin{array}{ccccccccccc}
0 & \cdots & 0 & \frac{x_{0} \varepsilon}{a-b} & 0 & \cdots & 0 & \frac{-x_{0} \varepsilon}{a-b} & 0 & \cdots & 0 \\
\vdots & \cdots & \vdots & \vdots & \vdots & \cdots & \vdots & \vdots & \vdots & \cdots & \vdots \\
0 & \cdots & 0 & \frac{x_{i} \varepsilon}{a-b} & 0 & \cdots & 0 & \frac{-x_{i} \varepsilon}{a-b} & 0 & \cdots & 0 \\
\vdots & \cdots & \vdots & \vdots & \vdots & \cdots & \vdots & \vdots & \vdots & \cdots & \vdots \\
0 & \cdots & 0 & \frac{x_{r} \varepsilon}{a-b} & 0 & \cdots & 0 & \frac{-x_{r} \varepsilon}{a-b} & 0 & \cdots & 0
\end{array}\right)
$$

is $\left\{\lambda_{0}, \lambda_{1}+x^{t} p, \lambda_{2}, \ldots, \lambda_{r}\right\}=\left\{\lambda_{0}, \lambda_{1}+\varepsilon, \lambda_{2}, \ldots, \lambda_{r}\right\}$. Note that the matrix $A+x p^{t}$ has constant row sums equal to $\lambda_{0}$. Second, the spectrum of $A+x p^{t}+e q^{t}$ is $\left\{\lambda_{0}+e^{t} q, \lambda_{1}+\varepsilon, \lambda_{2}, \ldots, \lambda_{r}\right\}=\Lambda_{\varepsilon}$.

Hence $\Lambda_{\varepsilon}$ satisfies Perfect $2^{+}$with $A_{\varepsilon}$ and the same partition of $\left\{\lambda_{r+1}, \ldots \lambda_{n}\right\}$ as $\Lambda$.

Case $\lambda_{1}=\lambda_{0}$. From Lemma 2 we can take $A$ as

$$
A=\left(\begin{array}{ccccc}
B_{11} & 0 & \cdots & \cdots & 0 \\
B_{21} & B_{22} & 0 & \cdots & 0 \\
\vdots & 0 & \ddots & \ddots & \vdots \\
\vdots & \vdots & \ddots & \ddots & 0 \\
B_{k 1} & 0 & \cdots & 0 & B_{k k}
\end{array}\right)
$$

with $\lambda_{0}$ eigenvalue of $B_{11}$ and $\lambda_{1}$ eigenvalue of some $B_{i i}$ with $i \geq 2$. Therefore, there exists an eigenvector $x$ of $A$ associated to $\lambda_{1}=\lambda_{0}$ linearly independent of $e$. Now we can apply the same argument and construction as in the case $\lambda_{1} \neq \lambda_{0}$.

e) $\Lambda_{\varepsilon}=\left\{\lambda_{0}+\varepsilon, \lambda_{1}, \ldots, \lambda_{r}, \lambda_{r+1}-\varepsilon, \lambda_{r+2}, \ldots, \lambda_{n}\right\}$ is Perfect $2^{+}$realizable.

Let $k \in\{0,1, \ldots, r\}$ such that $\lambda_{r+1} \in\left\{\lambda_{k 1}, \ldots, \lambda_{k t_{k}}\right\}$ and let $q$ be the vector with $\varepsilon$ in the position $k+1$ and the rest zeros. The matrix

$$
A_{\varepsilon}=A+e q^{t}=A+\left(\begin{array}{ccccccc}
0 & \cdots & 0 & \varepsilon & 0 & \cdots & 0 \\
\vdots & \ddots & \vdots & \vdots & \vdots & \ddots & \vdots \\
0 & \cdots & 0 & \varepsilon & 0 & \cdots & 0
\end{array}\right)
$$

is nonnegative with diagonal elements $\omega_{0}, \omega_{1}, \ldots, \omega_{k-1}, \omega_{k}+\varepsilon, \omega_{k+1}, \ldots, \omega_{r}$ and all its row sums equal to $\lambda_{0}+\varepsilon$. Brauer's theorem guarantees that the spectrum of $A_{\varepsilon}$ is $\left\{\lambda_{0}+e^{t} q, \lambda_{1}, \ldots, \lambda_{r}\right\}=\left\{\lambda_{0}+\varepsilon, \lambda_{1}, \ldots, \lambda_{r}\right\}$. Hence $\Lambda_{\varepsilon}$ satisfies Perfect $2^{+}$with $A_{\varepsilon}$ and the "same" partition of $\left\{\lambda_{r+1}-\varepsilon, \lambda_{r+2}, \ldots \lambda_{n}\right\}$ as $\Lambda$.

f) $\Lambda_{\varepsilon}=\left\{\lambda_{0}+\varepsilon, \lambda_{1}-\varepsilon, \lambda_{2}, \ldots, \lambda_{r}, \lambda_{r+1}, \ldots, \lambda_{n}\right\}$, with $\lambda_{1}-\varepsilon \geq 0$, is Perfect $2^{+}$realizable.

Let $x=\left(x_{0}, x_{1}, \ldots, x_{r}\right)^{t}$ be an eigenvector of $A$ associated to $\lambda_{1}$ linearly independent of $e$. The existence of this eigenvector was proved above. Let $j_{\max }, j_{\min }, a$ and $b$ be as before. Now let $q$ and $p$ be defined by

$$
q_{i}=\left\{\begin{array}{ll}
\frac{a \varepsilon}{a-b} & \text { if } \quad i=j_{\max }, \\
\frac{-b \varepsilon}{a-b} & \text { if } \quad i=j_{\min }, \\
0 & \text { if } \quad i \neq j_{\max }, j_{\min }
\end{array} \quad \text { and } \quad p_{i}= \begin{cases}\frac{-\varepsilon}{a-b} \quad \text { if } \quad i=j_{\max }, \\
\frac{\varepsilon}{a-b} \quad \text { if } \quad i=j_{\min }, \\
0 \quad \text { if } \quad i \neq j_{\max }, j_{\min } .\end{cases}\right.
$$


Note that the matrix $A_{\varepsilon}=A+e q^{t}+x p^{t}$

$$
A_{\varepsilon}=A+\left(\begin{array}{ccccccccccc}
0 & \cdots & 0 & \frac{\left(a-x_{0}\right) \varepsilon}{a-b} & 0 & \cdots & 0 & \frac{\left(x_{0}-b\right) \varepsilon}{a-b} & 0 & \cdots & 0 \\
\vdots & \cdots & \vdots & \vdots & \vdots & \cdots & \vdots & \vdots & \vdots & \cdots & \vdots \\
0 & \cdots & 0 & \frac{\left(a-x_{i}\right) \varepsilon}{a-b} & 0 & \cdots & 0 & \frac{\left(x_{i}-b\right) \varepsilon}{a-b} & 0 & \cdots & 0 \\
\vdots & \cdots & \vdots & \vdots & \vdots & \cdots & \vdots & \vdots & \vdots & \cdots & \vdots \\
0 & \cdots & 0 & \frac{\left(a-x_{r}\right) \varepsilon}{a-b} & 0 & \cdots & 0 & \frac{\left(x_{r}-b\right) \varepsilon}{a-b} & 0 & \cdots & 0
\end{array}\right)
$$

is nonnegative with diagonal elements $\omega_{0}, \omega_{1}, \ldots, \omega_{r}$ and all its row sums equal to $\lambda_{0}+\varepsilon$. Again, the application twice of Brauer's theorem guarantees that the spectrum of $A_{\varepsilon}$ is $\left\{\lambda_{0}+e^{t} q, \lambda_{1}+x^{t} p, \lambda_{2}, \ldots, \lambda_{r}\right\}=$ $\left\{\lambda_{0}+\varepsilon, \lambda_{1}-\varepsilon, \lambda_{2}, \ldots, \lambda_{r}\right\}$. Hence $\Lambda_{\varepsilon}$ verifies Perfect $2^{+}$with $A_{\varepsilon}$ and the same partition of $\left\{\lambda_{r+1}, \ldots \lambda_{n}\right\}$ as $\Lambda$. This shows that game implies Perfect $2^{+}$.

The list $\{6,1,1,-4,-4\}$ is not game realizable, see [3], but is Perfect $2^{+}$realizable because the matrix

$$
\left(\begin{array}{ccc}
4 & 0 & 2 \\
3 / 2 & 4 & 1 / 2 \\
0 & 6 & 0
\end{array}\right)
$$

realizes $\{6,1,1\}$ and the sum of the elements of each one of the lists $\{4,-4\},\{4,-4\}$ and $\{0\}$ is nonnegative. This shows the inclusion is strict.

Theorem 10. The inclusion of Perfect $2^{+}$, with $r \geq 1$, in Soto-Rojo, with $s \geq 2$, is strict.

Proof. The inclusion is clear. The list $\{8,6,5,5,-4,-4,-4,-4,-4,-4\}$ satisfies Soto-Rojo for the partition $\{8,5,-4,-4,-4\} \cup\{6,5,-4,-4,-4\}:\{8,6\}$ is realizable by a nonnegative matrix with diagonal elements 7 and 7 and $\{7,5,-4,-4,-4\}$ is realizable because it satisfies the necessary and sufficient conditions given in [9].

This list is not Perfect $2^{+}:$if $\{8,6,5,5\}$ is realized by $A=\left(a_{i j}\right)$ with diagonal elements $w_{0}, w_{1}, w_{2}$ and $w_{3}$, we have

$$
w_{0}+w_{1}+w_{2}+w_{3}=24, \text { and } 0 \leq w_{i} \leq 8 \text { for } i=0, \ldots, 3 \text {. }
$$

We can take $A$ with all its row sums equal to 8 , see Lemma 2 . Then the two possibilities to partition $\{-4,-4,-4,-4,-4,-4\}$ such that Perfect $2^{+}$is satisfied are

$$
\{-4,-4\} \cup\{-4,-4\} \cup\{-4,-4\} \cup \emptyset \Rightarrow\left\{\begin{array}{l}
\omega_{0}=\omega_{1}=\omega_{2}=8 \\
\omega_{3}=0
\end{array}\right.
$$

and

$$
\{-4,-4\} \cup\{-4,-4\} \cup\{-4\} \cup\{-4\} \Rightarrow\left\{\begin{array}{l}
\omega_{0}=\omega_{1}=8 \\
\omega_{3}=8-\omega_{2}
\end{array} .\right.
$$

The nonnegative character of $A$ implies, in both cases, that $A$ is of the form

$$
A=\left(\begin{array}{cccc}
8 & 0 & 0 & 0 \\
0 & 8 & 0 & 0 \\
* & * & * & * \\
* & * & * & *
\end{array}\right)
$$

which contradicts that the spectrum of $A$ is $\{8,6,5,5\}$. 
Remark 1. Note that the list $\{8,6,5,5,-4,-4,-4,-4,-4,-4\}$ given in the previous proof is not Perfect $2^{+}$but it satisfies Perfect 2, that is Theorem 1.

Recently, Ellard-Šmigoc [5], via a recursive approach to symmetric realizations, have established the equivalence of several of the most general sufficient conditions for symmetric realization. In particular, they prove the equivalence

$$
\text { C-realizability } \Longleftrightarrow \bigcup_{p \geq 2} \text { Soto } p \text {. }
$$

As a consequence we have that game and $C$-realizability are also equivalent, since

$$
\bigcup_{p \geq 2} \text { Soto } p \Rightarrow \text { game } \Rightarrow C \text {-realizability } \Longleftrightarrow \bigcup_{p \geq 2} \text { Soto } p \text {. }
$$

Next we summarize the previous results in a map and we give examples to explain it.

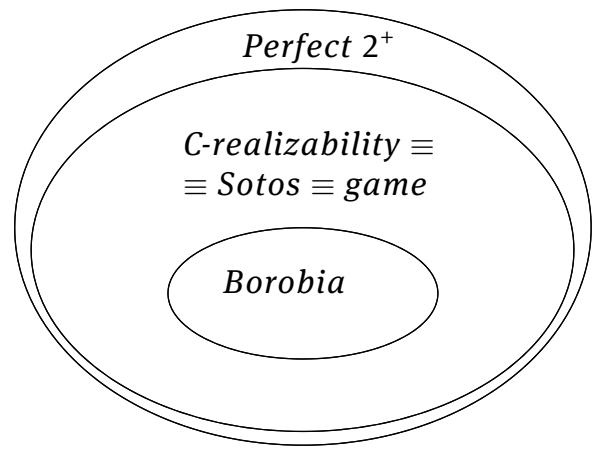

$$
\text { Sotos }=\bigcup_{p \geq 2} \text { Soto } p
$$

$\bar{X}=$ Condition $\mathrm{X}$ is not satisfied

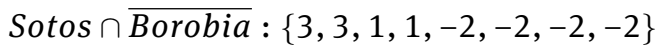

Perfect $2^{+} \cap \overline{\text { game }}:\{6,1,1,-4,-4\}$

Perfect $2^{+}$, the strongest criteria of the map, presents the difficulty that it requires the construction of an $(r+1)$-by- $(r+1)$ matrix with fixed diagonal realizing the given spectrum, which is an open problem.

Acknowledgement: Supported by Fondecyt 1170313, Chile; MTM2015-365764-C-1 (MINECO/FEDER); MTM2017-85996-R (MINECO/FEDER); Consejería de Educación de la Junta de Castilla y León (Spain) VA128G18.

We would like to express our deep gratitude to Charles Johnson for his many relevant and groundbreaking contributions to Mathematics. We would also like to think him for all the nice working times together.

\section{References}

[1] A. Borobia, On the Nonnegative Eigenvalue Problem, Linear Algebra Appl. 223-224 (1995) 131-140.

[2] A. Borobia, J. Moro, R. L. Soto, Negativity compensation in the nonnegative inverse eigenvalue problem, Linear Algebra Appl. 393 (2004) 73-89.

[3] A. Borobia, J. Moro, R. L. Soto, A unified view on compensation criteria in the real nonnegative inverse eigenvalue problem, Linear Algebra Appl. 428 (2008) 2574-2584.

[4] A. Brauer, Limits for the characteristic roots of a matrix, IV: Applications to stochastic matrices, Duke Math. J. 19 (1952) 75-91.

[5] R. Ellard, H. Šmigoc, Connecting sufficient conditions for the symmetric nonnegative inverse eigenvalue problem, Linear Algebra Appl. 498 (2016), 521-552.

[6] W. Guo, Eigenvalues of nonnegative matrices, Linear Algebra Appl. 266 (1997) 261-270.

[7] C. R. Johnson, Row stochastic matrices similar to doubly stochastic matrices, Linear Multilinear Algebra 10 (1981) 113-130.

[8] R. Kellogg, Matrices similar to a positive or essentially positive matrix, Linear Algebra Appl. 4 (1971) 191-204.

[9] T. J. Laffey and E. Meehan, A characterization of trace zero nonnegative $5 \times 5$ matrices, Linear Algebra Appl. $302 / 303$ (1999) 295-302.

[10] C. Marijuán, M. Pisonero, On Sufficient Conditions for the RNIEP and their Margins of Realizability, Electron. Notes Discrete Math. 46 (2014) 201-208. 
[11] C. Marijuán, M. Pisonero, R. L. Soto, A map of sufficient conditions for the real nonnegative inverse eigenvalue problem, Linear Algebra Appl. 426 (2007) 690-705.

[12] H. Perfect, Methods of constructing certain stochastic matrices II, Duke Math.J.22 (1955) 305-311.

[13] R. L. Soto, Existence and construction of nonnegative matrices with prescribed spectrum, Linear Algebra Appl. 369 (2003) 169-184.

[14] R. L. Soto, A family of realizability criteria for the real and symmetric nonnegative inverse eigenvalue problem, Numer. Linear Algebra Appl. 20 (2013) 336-348.

[15] R. L. Soto, O. Rojo, Applications of a Brauer theorem in the nonnegative inverse eigenvalue problem, Linear Algebra Appl. 416 (2006) 844-856.

[16] R. L. Soto, O. Rojo, C. B. Manzaneda, On Nonnegative Realization of Partitioned Spectra, Electron. J. Linear Algebra 22 (2011) 557-572. 